\title{
Myocardial Ischemia Diagnosis Using a Reduced Lead System
}

Citation for published version (APA):

Aranda Hernandez, A., Bonizzi, P., Karel, J., \& Peeters, R. (2018). Myocardial Ischemia Diagnosis Using a Reduced Lead System. In IEEE Engineering in Medicine and Biology Society. 2018 (pp. 5302-5305) https://doi.org/10.1109/EMBC.2018.8513511

Document status and date:

Published: 01/07/2018

DOI:

10.1109/EMBC.2018.8513511

Document Version:

Publisher's PDF, also known as Version of record

Document license:

Taverne

Please check the document version of this publication:

- A submitted manuscript is the version of the article upon submission and before peer-review. There can be important differences between the submitted version and the official published version of record.

People interested in the research are advised to contact the author for the final version of the publication, or visit the DOI to the publisher's website.

- The final author version and the galley proof are versions of the publication after peer review.

- The final published version features the final layout of the paper including the volume, issue and page numbers.

Link to publication

\footnotetext{
General rights rights.

- You may freely distribute the URL identifying the publication in the public portal. please follow below link for the End User Agreement:

www.umlib.nl/taverne-license

Take down policy

If you believe that this document breaches copyright please contact us at:

repository@maastrichtuniversity.nl

providing details and we will investigate your claim.
}

Copyright and moral rights for the publications made accessible in the public portal are retained by the authors and/or other copyright owners and it is a condition of accessing publications that users recognise and abide by the legal requirements associated with these

- Users may download and print one copy of any publication from the public portal for the purpose of private study or research.

- You may not further distribute the material or use it for any profit-making activity or commercial gain

If the publication is distributed under the terms of Article $25 \mathrm{fa}$ of the Dutch Copyright Act, indicated by the "Taverne" license above, 


\title{
Myocardial Ischemia Diagnosis Using a Reduced Lead System
}

\author{
A. Aranda Hernandez ${ }^{1,2}$, P. Bonizzi ${ }^{2}$, J. Karel ${ }^{2}$ and R. Peeters ${ }^{2}$
}

\begin{abstract}
This research presents a novel statistical model for diagnosing acute myocardial infarction (AMI). The model is based on features extracted from a reduced lead system consisting of a subset of three leads from the standard 12-lead ECG. We selected a set of relevant parameters commonly used in the clinical practice for ECG-based AMI diagnosis, namely ST elevation and T-wave maximum. We also selected features, not used in clinical practice, that were derived from vectorcardiography and computed on the reduced three-lead system (pseudo-VCG parameters). To validate the model, we used 104 patients coming from the Physionet STAFF III database which contains 12-lead ECG recordings at baseline and in coronary artery occlusion condition during angioplasty (PTCA). Results show that pseudo-VCG features are able to diagnose AMI slightly better than ST elevation and T-wave maximum features together (area under the ROC curve (AUC) 0.87 vs AUC 0.85). When combining pseudo-VCG features together with ST elevation, and T-wave maximum, the performance improved significantly (AUC 0.95, sensitivity $\mathbf{8 9 . 6} \%$ and specificity $82.7 \%$ ). Results indicate a potential for diagnosing AMI using the proposed reduced lead system and the selected set of features. We suggest its possible use for diagnosing AMI in long-term, ambulatory and home monitoring situations, allowing an earlier and faster diagnosis.
\end{abstract}

\section{INTRODUCTION}

Coronary heart diseases are one of the leading causes of death worldwide and the leading cause of mortality in America [1]. One of these coronary heart diseases is myocardial infarction, known as a heart attack, which is an occlusion of a coronary artery that results in an insufficient blood supply to the myocardium and damaging it.

Acute myocardial infarction (AMI), also called myocardial ischemia, is the initial step of myocardial infarction and results from an imbalance between oxygen supply and demand. Morbidity and mortality from AMI are significantly reduced when symptoms are recognized early enough, shortening time to treatment.

The diagnostic tests for AMI, commonly used in clinical practice, include ECGs, computer imaging, blood enzyme, chest X-ray, cardiac catheterization etc. Nonetheless, these tests are not only very expensive but also not always available.

The ECG is a routine part of the diagnostic work-up of patients with suspected AMI. The more common ECG manifestations of AMI (in absence of LVH and LBBB) are ST elevation, ST depression and T-wave changes [2]. The

${ }^{1}$ A. Aranda Hernandez is with the Department of Research and Technology in the Bakken Research Center, Medtronic, Maastricht, The Netherlands

${ }^{2}$ A. Aranda Hernandez, P. Bonizzi, J. Karel and R. Peeters are with the Department of Data Science and Knowledge Engineering, Maastricht University, Maastricht, The Netherlands. standard 12-lead ECG is characterized by a certain degree of redundancy, due to the fact that some of the leads are nearly aligned or derived as linear combinations of other leads. This redundancy already suggests the possibility to obtain reliable and accurate information from a subset of those leads. Recently, we used vectorcardiography (VCG) [3][4], to diagnose myocardial infarction [12], but not AMI. Different studies have focused on 12-lead ECG systems for diagnosing AMI [15]. However, a reduced set of ECG leads would be more convenient in situations of long-term, ambulatory, and home monitoring, making it possible an earlier diagnosis of AMI.

In this research, we introduce a novel statistical model for diagnosing AMI using a reduced set of three ECG leads. Although the reduced lead system we suggest is not comparable with conventional VCG, we used it to compute standard VCG features, and for this reason we refer to those as pseudo-VCG features. The best subset of three ECG leads for diagnosing AMI was found using the backward stepwise method. The reduced lead system was used to build three different models to diagnose AMI based on ST elevation and T-wave maximum criteria (model 1), pseudo-VCG features (model 2), and a combination of all these features (model 3), respectively. Model 3 gave best performance with AUC 0.95, sensitivity $89.6 \%$ and specificity $82.7 \%$.

\section{MATERIALS AND METHODS}

\section{A. Database}

We used the PhysioNet STAFF III database [5][6] as data source. This database consists of standard 12-lead ECG recordings from 104 patients with stenotic coronary arteries, who received elective percutaneous transluminal coronary angiography (PTCA) in one of the major coronary arteries, thus simulating AMI episodes in patients. The database documents the first few minutes of complete coronary occlusion.

The original objective of the database was to provide a better understanding of the manifestations of AMI in the ECG in respect to high-frequency components, particularly during ventricular depolarization [7]. From the 104 patients' recordings, 6 patients were excluded because of either problems in the 12-lead configuration or noise. We used two recordings per patient. The first one is a baseline recording preceding intervention and at rest. The second one is selected in the interval between catheter balloon inflation and deflation during the PTCA procedure. This second recording contains the coronary artery occlusion period and records the ischemic event.

As baseline (no AMI), 10 seconds of ECG data in the middle of the baseline recordings were selected. We used 10 seconds of ECG data in the occlusion period during PTCA as AMI recordings. In this last case, the recordings were noisier, 
so we decided to select the 10 seconds intervals by visual inspection to ensure the desired quality in the ECG signals. In average the 10 seconds interval was selected 181 seconds after the occlusion starts.

\section{B. Feature Extraction}

In order to characterize AMI, a set of features was derived from the reduced lead system, which can be classified in two groups:

1) $\mathbf{T}$-wave features. Namely $S T$ elevation and maximum in the T-wave. Since we have three ECG leads, a total of six features were computed.

2) Pseudo-VCG features. A total of 322 pseudo-VCG features were computed from the reduced lead system (see below for details).

The pseudo-VCG features that were calculated can be grouped into the following categories:

a) Geometrical Features (150 features) [8], computed for the QRS and T-wave loops: perimeter of the loop, centroid of the loop, maximum vector length, area of the loop, maximum distance from the centroid to the loop, angle of the loop with $\mathrm{XY}$ plane, angle of the loop with XZ plane, angle between QRS maximum vector length and $T$ wave maximum vector length, angle between QRS optimal plane and $\mathrm{T}$ wave optimal plane. We measured those features in different planes. For instance, the perimeter of the QRS or T-wave loop can be measure in the horizontal, frontal or sagittal planes as well as in the 3D space.

b) Spatial and Temporal Features (64 features) [9][10], computed for the QRS and T-wave loops: octant location of the loops, percentage of time of the vector in an octant, average and variance of the vector magnitude in each octant. Notice that there are eight octants and therefore eight parameters for each one of the described features. Also notice that we have calculated those parameters in the first derivative of the ECG signals as well.

c) Statistical Parameters (108 features): standard deviation, Shannon entropy, kurtosis, Hurst exponent, and skewness. Notice that these features are calculated for each of the ECG leads and that every feature is compose of different parameters. For instance, Hjorth feature is compose as well of Hjorth activity, mobility and complexity. Also notice that we have calculated as well those parameters in the first derivative of the ECG signals.

\section{Reduced Lead System Selection}

To select the best 3-lead ECG system, we used backward stepwise selection [11]. This was based on a model including all features introduced in Sec. II.B. In order to reduce overfitting, we divided the database into a training dataset with $70 \%$ of the patients (69 patients and 138 recordings) and a validation dataset with the other 30\% (29 patients and 58 recordings). Starting with the full set of 12-leads, backward stepwise selection iteratively removed one lead at time based on the AUC computed on the validation set, and by means of leave-one-out cross-validation.

The best three leads found for diagnosing AMI were V1, V4 and III, which were used to generate the reduced 3-lead ECG system.

\section{Feature Selection}

As mentioned before, we computed a set of 322 pseudoVCG features and six T-wave features. This high dimensional feature space is not efficient for classification in terms of computational performance. Also, having unnecessary features may add noise to the estimation of other quantities of interest and increase the chances of overfitting.

We used stepwise forward selection as method for variable selection, which in our analyses performed better than more advanced techniques such as lasso, ridge regression and random forest. We applied feature selection to both pseudo-VCG features and T-wave features. In the case of pseudo-VCG features, dimensionality was reduced from 322 to five features, while in the case of T-wave features it was reduced from six features to two features. Table I shows the five final pseudo-VCG features selected and Table II shows the two final T-wave features selected.

TABLE I. REDUCED SET OF PSEUDO-VCG FEATURES.

\begin{tabular}{|c|l|}
\hline Feature & \multicolumn{1}{c|}{ Description } \\
\hline T_PA_SP & $\begin{array}{l}\text { T-wave loop perimeter/area ratio in the sagittal } \\
\text { plane. }\end{array}$ \\
\hline T_AREA_SP & T-wave loop area in the sagittal plane. \\
\hline T_STD_Z & T-wave standard deviation in $z$ VCG component. \\
\hline QRS_PA_3D & QRS loop 3D perimeter/area ratio. \\
\hline QRS_AVG_X & QRS average vector length of VCG $x$ component. \\
\hline
\end{tabular}

TABLE II. REDUCED SET OF T-WAVE FEATURES.

\begin{tabular}{|c|c|}
\hline Feature & Description \\
\hline STE_III & ST elevation in lead III. \\
\hline STE_V4 & ST elevation in lead V4. \\
\hline
\end{tabular}

\section{E. Model Elaboration}

Using the reduced 3-lead ECG system described in section II.C, and the set of features described in Table I and Table II, we built three models for diagnosing AMI. All models were built by using the gradient boosting method implemented in the "gbm" $\mathrm{R}$ package [13]. Table III describes the three models that were created.

TABLE III. MODELS FOR DIAGNOSING AMI.

\begin{tabular}{|c|l|}
\hline Model & \multicolumn{1}{c|}{ Description } \\
\hline 1 & $\begin{array}{l}\text { Built using the T-wave features obtained after feature } \\
\text { selection (Table II). }\end{array}$ \\
\hline 2 & $\begin{array}{l}\text { Built using the pseudo-VCG features obtained after feature } \\
\text { selection (Table I). }\end{array}$ \\
\hline 3 & $\begin{array}{l}\text { Built using T-wave features and pseudo-VCG features } \\
\text { obtained after feature selection (Table II and Table I). }\end{array}$ \\
\hline
\end{tabular}

In order to properly train and validate the model, we divided the database into the same training and validation dataset introduced in Sec. II.C. The selection of the training and validation patients was done randomly over the 98 patients. We built each model using the training dataset and used the validation dataset to test the performance of the model. All the results presented in this work were obtained using the validation dataset. 


\section{F. AMI diagnosis based on location}

We were also interested in analyzing the variation of performance of the best model in diagnosing AMI depending on where AMI occurs.

Table IV shows how many recordings correspond to each of the AMI locations in the complete dataset. As it can be observed, there are few recordings for some of the AMI locations (e.g., LAD diagonal, Left main). Therefore, we grouped all the individual locations in three main regions, namely anterior, inferior, and posterior (first column in Table IV).

It can be observed that after grouping, the percentage of recordings corresponding to each AMI region is similar in the complete dataset and in the validation dataset. This shows that the validation dataset is representative for the entire dataset and the results obtained for this dataset can be generalized.

TABLE IV. AMI HEART REGIONS.

\begin{tabular}{|c|c|c|c|c|c|}
\hline AMI Region & AMI Location & $\begin{array}{c}N \\
\text { Complete }\end{array}$ & $\begin{array}{c}N \\
\text { Validation }\end{array}$ & $\begin{array}{c}\% \\
\text { Complete }\end{array}$ & $\begin{array}{c}\% \\
\text { Validation }\end{array}$ \\
\hline \multirow{5}{*}{ Anterior } & LAD diag & 4 & 2 & \multirow{5}{*}{$34 \%$} & \multirow{5}{*}{$31 \%$} \\
\hline & Left main & 4 & 0 & & \\
\hline & Mid LAD & 12 & 4 & & \\
\hline & Prox mid LAD & 6 & 4 & & \\
\hline & Prox LAD & 42 & 8 & & \\
\hline \multirow{4}{*}{ Inferior } & Mid RCA & 28 & 10 & \multirow{4}{*}{$45 \%$} & \multirow{4}{*}{$52 \%$} \\
\hline & Dist RCA & 22 & 12 & & \\
\hline & Prox RCA & 34 & 6 & & \\
\hline & Prox mid RCA & 4 & 2 & & \\
\hline \multirow{3}{*}{ Posterior } & Dist circ & 12 & 4 & \multirow{3}{*}{$21 \%$} & \multirow{3}{*}{$17 \%$} \\
\hline & Mid circ & 10 & 2 & & \\
\hline & Prox circ & 20 & 4 & & \\
\hline
\end{tabular}

\section{RESULtS}

Figure 1 shows the performance of each of the 3 models when using the validation dataset which includes data from 29 patients (30\% of the samples). Model 3 (combined features) gave the best performance with AUC 0.95, followed by model 2 (VCG features; AUC 0.87), and model 1 (ST elevation and T-wave maximum features; AUC 0.85).

We looked to significant differences in ROC curves values using DeLong's test for two correlated ROC curves and using a cut-off $\mathrm{p}$-value of $5 \%$. Table $\mathrm{V}$ shows the results when comparing the three models.

TABLE V. DELONG'S TEST FOR AUC FOR MODELS 1 TO 3.

\begin{tabular}{|c|c|}
\hline Models & p-value \\
\hline 1 vs 2 & 0.738 \\
\hline 2 vs 3 & 0.025 \\
\hline 1 vs 3 & 0.045 \\
\hline
\end{tabular}

When comparing model 1 with model 2 , the increase in AUC was not significant (0.85 vs. 0.87 , p-value 0.738$)$. When comparing model 2 with model 3 the difference in AUC was significant (0.87 vs. 0.95, p-value 0.025$)$. Also, the increase in AUC when comparing models 1 and 3 was significant $(0.85$ vs. 0.95 , p-value 0.045$)$. Based on these results, we decided to use model 3 as our best model for diagnosing AMI. In this model, we selected a cut-off value in the ROC curve using the training dataset. Using this cut-off value, the sensitivity and specificity in the validation dataset was $89.6 \%$ and $82.7 \%$ respectively.

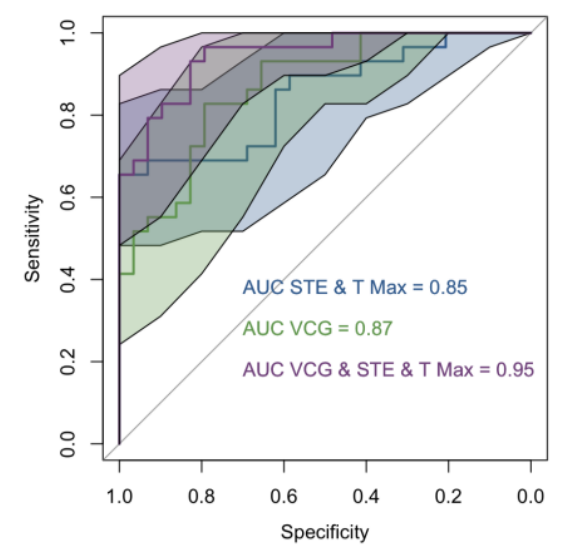

Figure 1. Performance of the three models on the validation dataset.

Figure 2 shows the performance of model 3 in the different heart regions (in the validation dataset) while Table VI shows the significance of the differences in AUC when diagnosing different AMI locations. As can be seen in Table VI, there were no significant differences in AUC when diagnosing AMI in the different heart regions.

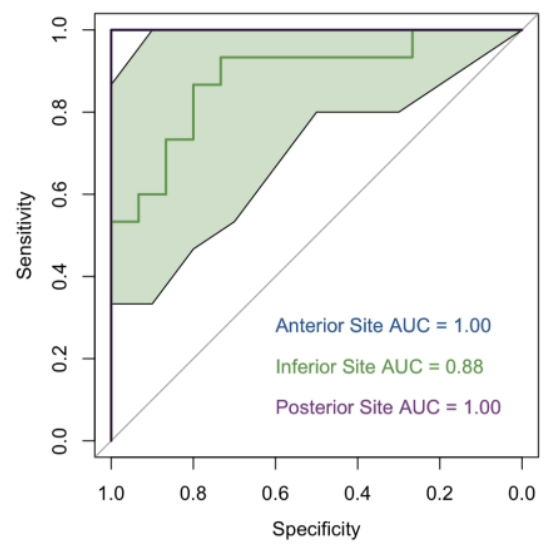

Figure 2. Model performance in the different AMI locations. Notice that purple and blue lines overlap because AUC is 1.00 for both.

TABLE VI. DELONG'S TEST FOR AUC FOR HEART REGIONS.

\begin{tabular}{|c|c|}
\hline Heart Regions & p-value \\
\hline Anterior vs Inferior & 0.076 \\
\hline Anterior vs Posterior & 1.000 \\
\hline Inferior vs Posterior & 0.076 \\
\hline
\end{tabular}

\section{DISCUSSION}

The main motivation of this study is the lack of a reduced lead system to diagnose AMI from the ECG. The results we presented suggests that it is possible to do a reliable diagnosis of AMI using a 3-lead ECG system. We observed that when 
clinical features were combined with pseudo-VCG features (model 3), the AUC significantly increased up to 0.95 vs AUC of 0.85 for T-wave features alone (model 1), and AUC of 0.87 for pseudo-VCG features alone (model 2). This may indicate that pseudo-VCG features have additional diagnostic relevant information not present in the clinical features. Indeed, Figure 1 shows that clinical features are more sensitive and less specific than pseudo-VCG features and that they complement each other. This match with actual literature on ST elevation that points out its high sensitivity and poor specificity. In the case of pseudo-VCG features we have both QRS and T-wave parameters, what we believe improves specificity by considering also the QRS and not only the T-wave.

When looking at the performance of model 3 in the different heart regions, the dubious high performance achieved in the anterior and posterior sites (AUC 1.00) compared to the inferior sites (AUC 0.88) may be due to the small number of patients in the anterior ( 9 patients) and posterior ( 5 patients) regions, compared to the inferior region (15 patients). Interestingly, we observe that the model is able to diagnose AMI in the posterior part of the heart. Patients having posterior MI (PMI) cannot currently be identified using simple ECG analysis [14]. The reason according to some researchers is that there are no specific leads in the standard ECG that represents this area. In addition, the lack of ST-segment elevation (as seen in typical ST-elevation MI) combined with misinterpreting the anterior ST-segment depressions as indicating ischemia rather than posterior infarction, frequently lead to missing PMI [14].

A consideration to keep in mind is that the patient population used in this research is at rest and not doing exercise. Being able to diagnose AMI at rest facilitates the diagnosis process of AMI, no longer requiring an exercise tolerance tests (ETT). This simplifies the diagnosis AMI and together with having a reduced lead system makes this solution suitable for long-term, remote, and home monitoring.

One limitation of this research is that we used a database in which we have an artificial AMI produced by the occlusion of one of the coronary arteries during the PTCA procedure. We aim to validate those results with more data coming from AMI patients. Also, we aim to confirm the suitability of this method to diagnose Non-ST-elevation myocardial ischemic patients. Another limitation of this study was the relatively small sample size. The results we reported need to be confirmed on a larger dataset.

\section{CONCLUSIONS}

The diagnostic tests for AMI, commonly used in clinical practice, include ECGs, computer imaging, blood enzyme, chest X-ray, cardiac catheterization etc. Nonetheless, these tests are not only very expensive but also not always available. This is especially true for long-term, ambulatory, and remote monitoring situations.

We showed that it may be possible to achieve a reliable diagnosis of AMI using a 3-lead ECG system composed of leads V1, V4 and III. We believe this simplification is especially important and meaningful in the long-term, remote, and home monitoring scenarios. The model we proposed uses T-wave and pseudo-VCG features and was able to diagnose AMI with $89.6 \%$ sensitivity and $82.7 \%$ specificity on a dataset with induced/simulated AMI events by PTCA procedure. The proposed method was also able to diagnose AMI in the posterior part of the heart. This, suggests its potential use for automatic detection of AMI from ECGs of PMI patients, for which diagnosis from ECGs is difficult nowadays.

\section{ACKNOWLEDGMENT}

The authors gratefully acknowledge Medtronic's Coronary Structural Heart Group for the partial funding of this research.

\section{REFERENCES}

[1] WRITING GROUP MEMBERS, Benjamin, E. J., Blaha, M. J., Chiuve, S. E., Cushman, M., Das, S. R., Muntner, P. (2017). Heart Disease and Stroke Statistics-2017 Update: A Report From the American Heart Association. Circulation, 135(10), e146-e603.

[2] Thygesen, K., Alpert, J. S., Jaffe, A. S., Simoons, M. L., Chaitman, B. R., White, H. D., Wagner, D. R. (2012). Third universal definition of myocardial infarction. Circulation, 126(16), 2020-2035.

[3] Frank, E. (1956). An accurate, clinically practical system for spatial vectorcardiography. Circulation 13:737.

[4] Boutkan, J. (1965). Vectorcardiography: physical bases and clinical practice. First ed. Eindhoven: Centrex Publishing Company.

[5] Pettersson, J., Carro, E., Edenbrandt, L., Pahlm, O., Ringborn, M., Srnmo, L., Warren, S., Wagner, G. (2000). Spatial, individual and temporal variation of the high frequency QRS amplitudes in the 12 standard electrocardiographic leads. Am Heart J, 139:352-358.

[6] Goldberger, A., Amaral, L., Glass, L., Hausdorff, J., Ivanov, P., Mark, R., et al. (2000). PhysioBank, PhysioToolkit, and PhysioNet: components of a new research resource for complex physiologic signals. Circulation.

[7] Martínez JP, Pahlm O, Ringborn M, Warren S, Laguna P, Sörnmo L. (2017) The STAFF III Database: ECGs Recorded During Acutely Induced Myocardial Ischemia. Comput Cardiol, 44:266-133.

[8] Correa, R., Arini, P. D., Valentinuzzi, M. E., Laciar, E. (2013 January) Novel set of vectorcardiographic parameters for the identification of ischemic patients. Med Eng Phys, 35(1), 16-22.

[9] Yang, H., Bukkapatnam, S.T.S., Le, T., Komanduri, R. (2012 May). Identification of myocardial infarction (MI) using spatio-temporal heart dynamics. Med Eng Phys, 34(4):485-97.

[10] Laufberger, V. (1980) Octant vectorcardiography. Physiol Bohemoslov, 29(6):481-94.

[11] Hocking, R. R. (1976). The Analysis and Selection of Variables in Linear Regression, Biometrics, 32.

[12] Aranda A., Bonizzi P., Karel J., Peeters, R. (2015). Performance of Dower's Inverse Transform and Frank Lead System for Identification of Myocardial Infarction. Engineering in Medicine and Biology Society (EMBC), 2015 37th Ann. Int. Conf. of the IEEE. pp. 44954498.

[13] Greg Ridgeway with contributions from others (2017). gbm: Generalized Boosted Regression Models. R package version 2.1.3. https://CRAN.R-project.org/package $=$ gbm.

[14] Levis, J. T. (2015). ECG Diagnosis: Isolated Posterior Wall Myocardial Infarction. The Permanente Journal, 19(4), e143-e144.

[15] Herring, N., \& Paterson, D. J. (2006). ECG diagnosis of acute ischaemia and infarction: Past, present and future. QJM - Monthly Journal of the Association of Physicians, 99(4), 219-230.

[16] Menown, I.B., Mackenzie, G., Adgey, A.A. (2002). Optimizing the initial 12-lead electrocardiographic diagnosis of acute myocardial infarction. Eur Heart J, 1:275-83.

[17] Treskes, R. W., Ter Haar, C. C., Man, S., De Jongh, M. C., Maan, A. C., Wolterbeek, R., Swenne, C. (2015). Performance of ST and ventricular gradient difference vectors in electrocardiographic detection of acute myocardial ischemia. Journal of Electrocardiology, 48(4), 498-504. 